\title{
Selective DF Relaying in Multi-Relay Networks With Different Modulation Levels
}

\author{
Hamza Umit Sokun*, Akram Bin Sediq*, Salama Ikki ${ }^{\dagger}$, and Halim Yanikomeroglu* \\ *Department of Systems and Computer Engineering, Carleton University, Ottawa, Ontario, Canada \\ ${ }^{\dagger}$ Department of Electrical Engineering, Lakehead University, Thunder Bay, Ontario, Canada \\ E-mail: \{husokun, akram, halim\}@ sce.carleton.ca, sikki@lakeheadu.ca
}

\begin{abstract}
Despite the rich literature on cooperative networks, employment of different modulation levels by the source and relay terminals has not been investigated thoroughly from the physical layer perspective. In this paper, we investigate the bit error rate (BER) performance of selective relaying in a multi-relay decodeand-forward cooperative network where the source and the relays transmit using different modulation levels. Specifically, we derive a closed form expression for the end-to-end (uncoded) BER. To draw further insights on the BER performance, we also provide a simpler approximate BER expression that is accurate in the high signal-to-noise ratio regime. Finally, simulation results are presented to verify the analytical results.

The derived BER expressions can be utilized in various other scenarios in which the destination selects the best signal (in terms of minimizing BER) among a set of signals which use different modulation levels. The set of signals to choose from may have already been received through orthogonal channels (selection combining), or this signal set may correspond to a set of "candidate" transmissions. The latter scenario is often referred to as selective transmission; applications of this scenario include selective relaying (the setting in this paper), fast base-station selection, and coordinated multipoint transmission and reception (CoMP).
\end{abstract}

\section{INTRODUCTION}

The demand for reliable high-speed wireless networks increases on a daily basis due to the augmentation of a wide range of wireless multimedia and interactive internet services. One of the promising technologies to enhance the performance of wireless networks is cooperative relaying, where the source communicates with the destination through one or more intermediate relays. In cooperative relaying, the decoding reliability can be substantially improved through the spatial diversity achieved at the destination by combining the signals received from the source and the relays.

In most of the multi-relay cooperative diversity protocols considered in the literature, relays perform retransmissions in orthogonal channels; as a consequence, spatial diversity is attained at the expense of end-to-end (e2e) spectral efficiency. Clearly, in order to have a favourable diversity versus e2e spectral efficiency trade-off, the retransmissions have to be made in a selective manner. As such, selective relaying has become a popular protocol in cooperative multi-relay literature in recent years [1].

This work is supported in part by Huawei Canada Co., Ltd., and in part by the Ontario Ministry of Economic Development and Innovation's ORF-RE (Ontario Research Fund - Research Excellence) program.
In selective relaying, adaptive modulation can be utilized to improve the e2e spectral efficiency. In [2], the authors investigate the performance of adaptive modulation in selective amplify-and-forward (AF) relaying networks. It is worth noting that in AF relaying, modulation adaptation is a possibility only at the source. Alternatively, in decode-and-forward (DF) relaying, both source and relays can employ different modulation levels opportunistically to further improve the e2e spectral efficiency, since these transmitting nodes may be experiencing different path loss and fading conditions [3]-[8].

Techniques to combine signals with different modulation levels have been considered in the literature for a long time in frameworks other than relaying (such as HARQ); a number of such techniques are part of the contemporary wireless standards. These techniques can readily be extended to relay networks as well. However, the overwhelming majority of these techniques have been in the form of post-detection combining, such as Chase Combining and Packet Selection Combining [3], [4]. The focus of this paper, on the other hand, is pre-detection combining which has a number of advantages over postdetection combining such as simplicity. While pre-detection combining of signals with the same modulation level has a long history itself in communications theory, the pre-detection combining of signals with different modulation levels arises as a rather new problem in the literature. This is due to the fact that transmission with different modulation levels implies an underlying distributed radio access network with cooperative transmission - a more recent framework.

The pre-detection combining techniques can be divided into two general types. In the first type, the signals are "mixed"; maximum ratio combining (MRC) is an example of this type. In the second type called selection combining (SC), on the other hand, the best signal is chosen rather than mixing them; SC has the obvious advantage of reduced complexity (at the expense of some performance loss).

In [5] and [6], the pre-detection combining of signals with different modulation levels in the form of mixing is studied. In [5], a combining scheme based on log-likelihood ratios (LLR), which rely on the maximum a posteriori probability principle, is proposed. In [6], another LLR-based combining scheme, which relies on the so-called "soft-bit MRC" principle is presented; it is shown that the performance of this scheme is very close to that of the optimal maximum likelihood detector.

It is well known that the selection combining of signals with 
the same modulation level means choosing the signal with the highest SNR; let us call this scheme SNR-SC. To the best of our knowledge, [7] and [8] are the first papers that generalized the operation of selection combining to include the signals with different modulation levels. It should be noted that, when the modulation levels are different, selecting the signal with the highest SNR will not be optimal any more due to the fact that the error resilience of those signals will be different. Instead, the selection in [7] and [8] is performed based on minimizing the BER (therefore the name, BER-SC). In the final analysis, BER-SC is also an SNR-based scheme where the selection is made among the appropriately biased (weighted) SNR values.

The analyses presented in [7] and [8] are for single-relay DF networks. In this paper, we investigate the performance of selective relaying in multi-relay DF networks when the source and relays use different modulation levels (the same modulation level at the source and relays is a special case of this more generic scenario). In the considered transmission scheme, the source communicates with the destination via the direct link or the best relayed link, based on the comparison of the instantaneous SNR values at the destination. The link that will yield the highest biased SNR value, which will minimize the BER at the destination, is identified. This link will be either the direct link (no relaying is necessary in that case), or one of the relayed links; in the latter case, only that particular relay will be requested to relay the original signal (i.e., selective relaying).

We derive a closed-form expression for the BER for the above explained scenario. We considered an uncoded system to isolate the BER expression from the parameters of any particular channel coding scheme. We also note that the uncoded packet error rate (PER) expressions can readily be obtained from the developed uncoded BER expressions. Finally, to draw further insights on the BER performance, we also provide a simpler approximate BER expression that is accurate in the high SNR regime.

The derived BER expressions can be utilized in various other scenarios in which the destination selects the best signal (in terms of minimizing BER) among a set of signals which use different modulation levels. The set of signals to choose from may have already been received through orthogonal channels (selection combining), or this signal set may correspond to a set of "candidate" transmissions. The latter scenario is often referred to as selective transmission; applications of this scenario include selective relaying (the setting in this paper), fast base-station selection, and coordinated multipoint transmission and reception (CoMP).

\section{SySTEM MODEL}

We consider a network that consists of a source $S$, a destination $D$, and $K$ relays $\left\{R_{k} \mid k=1, \ldots, K\right\}$. We assume that each terminal is equipped with a single antenna and operates in the half-duplex mode, i.e., it transmits and receives in orthogonal channels. Without loss of generality, we assume in this paper that orthogonality is achieved using time division duplexing in two time slots. In the first time slot, the source broadcasts an $N$-bit packet to the destination and the relays by using $M_{S}$-QAM. All the relays decode the received packet from the source, and check whether the packet is decoded correctly or not via cyclic redundancy codes (CRC). Then, in the second time slot, the relays that received the packet correctly form the decoding set $(\mathcal{D S})$ of candidate relays; each relay in the $\mathcal{D S}$ transmit a pilot signal and the modulation level to the destination. The destination estimates the SNRs from the source and from the relays in the $\mathcal{D S}$. Based on the SNRs and the modulation level of the source, $M_{S}$, and the modulation levels of the relays in the $\mathcal{D} \mathcal{S},\left\{M_{R_{k}} \mid k \in \mathcal{D} \mathcal{S}\right\}$, the destination chooses either to decode directly from the source or from one of the relays in the $\mathcal{D S}$. If the destination chooses to decode from the source, then it sends a one-bit negative acknowledgement to the $\mathcal{D S}$; otherwise, it asks the chosen relay to transmit the packet.

All links are assumed to experience Rayleigh fading. The instantaneous SNRs in the links $S-D, S-R_{k}$, and $R_{k}-D$ are denoted by $\gamma_{S D}, \gamma_{S R_{k}}$, and $\gamma_{R_{k} D}$, respectively, and they are independent exponential random variables. In addition, the average SNRs in the links $S-D, S-R_{k}$, and $R_{k}-D$ are denoted by $\bar{\gamma}_{S D}, \bar{\gamma}_{S R_{k}}$, and $\bar{\gamma}_{R_{k} D}$, respectively.

As stated earlier, BER-based selection scheme is better for different modulation levels as compared to SNR-based selection scheme since it accounts for the error-resistance capabilities of the different modulation levels. Therefore, we focus our attention on the performance of BER-based selection scheme. According to BER-based selection, the decision rule can be stated as

$$
\begin{cases}\text { select node } \mathrm{S}, \quad \text { if } \mathrm{BER}_{S D} \leq \mathrm{BER}_{R_{i} D}, \forall i \in \mathcal{D S} \\ \text { select node } \mathrm{R}_{i}, \quad \text { if } \mathrm{BER}_{S D}>\mathrm{BER}_{R_{i} D} \text { and } \\ \mathrm{BER}_{R_{j} D}>\mathrm{BER}_{R_{i} D}, j \neq i, \forall i, j \in \mathcal{D} \mathcal{S} .\end{cases}
$$

To simplify the selection criterion given in (1), we use the following approximate BER expression for Gray-coded square $M$-QAM [9]:

$$
\operatorname{BER}_{M_{i}}\left(\gamma_{i D}\right) \approx c_{M_{i}} Q\left(\sqrt{2 d_{M_{i}}^{2} \gamma_{i D}}\right),
$$

where

$$
\left(c_{M_{i}}, d_{M_{i}}\right)=\left\{\begin{array}{cl}
(1,1), & M_{i}=2, \\
\left(\frac{2-2 / \sqrt{M_{i}}}{\log _{2} \sqrt{M_{i}}}, \sqrt{\frac{3}{2\left(M_{i}-1\right)}}\right), & M_{i} \geq 4 .
\end{array}\right.
$$

All the derived equations can be used for any modulation scheme that has instantaneous BER in the form of $c_{M_{i}} Q\left(\sqrt{2 d_{M_{i}}^{2} \gamma_{i D}}\right)$.

\section{ERror Rate Performance AnAlysis}

In a relay-based cooperative communication implementation, packet-by-packet forwarding is used (one may argue for special scenarios for bit-by-bit forwarding, for instance, in some sensor networks; but these are exceptions rather than the norm). Therefore, in cooperative communication systems, the most appropriate metric is PER, rather than BER. While the e2e PER curves for any given protocol can always be obtained through 
simulations, the mathematical analysis of such scenarios is either very cumbersome, or not possible at all. Therefore, we present for a e2e BER analysis in this paper. The aim is to have some mathematical analysis that will reveal certain aspects of the complex relation between the essential dynamics of the system*.

In this section, we derive a closed form expression for the average BER in the multi-relay scenario. The average BER can written as

$$
\begin{aligned}
& \mathrm{BER}=\left(\prod_{k=1}^{K} \mathrm{PER}_{S R_{k}}\right) \mathrm{BER}_{S D} \\
& +\sum_{r=1}^{K} \sum_{m=1}^{\left|P_{r}\left(\mathcal{S}_{\text {all }}\right)\right|}\left[\left(\prod_{e_{i} \in P_{r, m}\left(\mathcal{S}_{\text {all }}\right)}\left(1-\mathrm{PER}_{S R_{e_{i}}}\right)\right)\right. \\
& \times\left(\prod_{e_{o} \notin P_{r, m}\left(\mathcal{S}_{\text {all }}\right)} \mathrm{PER}_{S R_{e_{o}}}\right) \mathrm{BER}_{\left.c o m p_{P_{r, m}\left(\mathcal{S}_{\text {all }}\right)}\right],}
\end{aligned}
$$

where

- $\mathcal{S}_{\text {all }}$ is the set of indices for all relays, i.e., $\mathcal{S}_{\text {all }}=\{1, \ldots, K\}$,

- $P_{r}\left(\mathcal{S}_{\text {all }}\right)$ is the $r$-element power set of $\mathcal{S}_{\text {all }}$,

- $P_{r, m}\left(\mathcal{S}_{\text {all }}\right)$ is the $m$-th element of $P_{r}\left(\mathcal{S}_{\text {all }}\right)=$ $\left\{P_{r, 1}\left(\mathcal{S}_{\text {all }}\right), \quad P_{r, 2}\left(\mathcal{S}_{\text {all }}\right), \quad \ldots \quad, P_{r,\left|P_{r}\left(\mathcal{S}_{\text {all }}\right)\right|}\left(\mathcal{S}_{\text {all }}\right)\right\}$,

- $\left|P_{r}\left(\mathcal{S}_{\text {all }}\right)\right|$ represents the cardinality of $P_{r}\left(\mathcal{S}_{\text {all }}\right)$,

- PER $R_{S k}$ is the average packet error rate in $S-R_{k}$ link,

- $\mathrm{BER}_{S D}$ is the average BER in $S-D$ link,

- $\mathrm{BER}_{\text {comp }_{\mathcal{D S}}}$ is average BER conditioned on the $\mathcal{D S}$ at destination.

For example, for a two-relay scenario,

$$
\begin{aligned}
& \mathrm{BER}=\mathrm{PER}_{S R_{1}} \mathrm{PER}_{S R_{2}} \mathrm{BER}_{S D} \\
& +\left(1-\mathrm{PER}_{S R_{1}}\right) \mathrm{PER}_{S R_{2}} \mathrm{BER}_{c o m p_{\{1\}}} \\
& +\left(1-\mathrm{PER}_{S R_{2}}\right) \mathrm{PER}_{S R_{1}} \mathrm{BER}_{c o m p_{\{2\}}} \\
& +\left(1-\mathrm{PER}_{S R_{2}}\right)\left(1-\mathrm{PER}_{S R_{1}}\right) \mathrm{BER}_{\text {comp }_{\{1,2\}}} .
\end{aligned}
$$

The average BER in the link from node $i$ to $j$ for $M$ QAM in a point-to-point Rayleigh fading channel can be well approximated as

$$
\begin{aligned}
\mathrm{BER}_{i j} & \approx \int_{0}^{\infty} c_{M_{i}} Q\left(\sqrt{2 d_{M_{i}}^{2} \gamma_{i j}}\right) \frac{1}{\bar{\gamma}_{i j}} e^{-\frac{\gamma_{i j}}{\bar{\gamma}_{i j}}} \\
& =\frac{1}{2} c_{M_{i}}\left(1-\sqrt{\frac{d_{M_{i}}^{2} \bar{\gamma}_{i j}}{1+d_{M_{i}}^{2} \bar{\gamma}_{i j}}}\right) .
\end{aligned}
$$

\footnotetext{
*An implementation note: Since we combine signals with different modulation levels, combining cannot be done on a bit-by-bit basis; rather combining should be done in units multiple of $L C M\left\{\log _{2}\left(M_{S}\right), \ldots ., \log _{2}\left(M_{R_{k}}\right)\right\}$ bits where $L C M$ represents the least common multiple. For example, if the source uses 16-QAM, and the relay uses 64-QAM, then combining at the receiver happens every 12 bits (or multiple of 12 bits); that is, the receiver combines 3 symbols from the source and 2 symbols from the relay. In other words, the receiver cannot operate in blocks less than 12 bits. In order not to lose tractability in the analysis, we do not take into account this implementation point.
}

If symbol errors occur independently an $N$-bit packet, then

$$
\begin{aligned}
& \operatorname{PER}_{S R_{i}}=1-\left(1-\mathrm{SER}_{S R_{i}}\right)^{\frac{N}{\log _{2} M_{s}}} \\
& \approx 1-\left(1-\frac{1}{2} c_{M_{s}} \log _{2}\left(M_{s}\right)\left(1-\sqrt{\frac{d_{M_{s}}^{2} \bar{\gamma}_{S R_{i}}}{1+d_{M_{s}}^{2} \bar{\gamma}_{S R_{i}}}}\right)\right)^{\frac{N}{\log _{2} M_{s}}}
\end{aligned}
$$

where SER $\approx \mathrm{BER} \log _{2} M_{s}$ for Gray-coded constellations [9].

The calculation of $\mathrm{BER}_{\text {comp }}$ is given in Section III-A, and the development of the average BER is given in Section III-B.

\section{A. Calculation of $B E R_{\text {comp }}$}

In the considered transmission scheme, the destination decodes the message from the source if the SNR of the source is larger than the biased SNRs of all the relays in $\mathcal{D S}$, i.e., $\gamma_{S D}=\max \left(\gamma_{S D}, \rho_{1} \gamma_{R_{1} D}, \ldots, \rho_{K} \gamma_{R_{K} D}\right)$, where $\rho_{i}=\frac{d_{M_{R_{i}}}^{2}}{d_{M_{S}}^{2}}$ is a biasing factor between the source and the relays. Otherwise, if $\gamma_{R_{i} D}=\max \left(\frac{1}{\rho_{i}} \gamma_{S D}, \frac{1}{\beta_{i 1}} \gamma_{R_{1} D}, \ldots, \frac{1}{\beta_{i K}} \gamma_{R_{K} D}\right)$, where $\beta_{i j}=\frac{d_{M_{R_{i}}}^{2}}{d_{M_{R_{j}}}^{2}}, i, j=1,2, \ldots, K$, is a biasing factor among the relays, then the $R_{i}-D$ link is chosen. Similar to [8], $\rho_{i}$ and $\beta_{i j}$ are introduced here in order to approximate BER-based selection in the high SNR regime. Such an approximation facilitates the BER analysis. Note that if all nodes transmit their signal with the same modulation level, then, BER-based selection scheme will be reduced to SNR-based selection scheme, i.e., $\rho_{i}=\beta_{i j}=1$.

An approximate and simpler implementation of the instantaneous BER for our scheme given by (1) can be expressed as

$$
\begin{aligned}
& \text { BER }_{\text {comp }, \text { inst }} \approx \\
& \begin{cases}c_{M_{S}} Q\left(\sqrt{2 d_{M_{S}}^{2} \gamma_{S D}}\right), & \gamma_{S D} \geq \rho_{i} \gamma_{R_{i} D}, \\
& \\
c_{M_{R_{i}}} Q\left(\sqrt{2 d_{M_{R_{i}}}^{2} \gamma_{R_{i} D}}\right), & \gamma_{S D}<\rho_{i} \gamma_{R_{i} D}, \text { and } \\
\gamma_{R_{j} D}<\beta_{i j} \gamma_{R_{i} D}, \\
j \neq i, j=1, \ldots, K .\end{cases}
\end{aligned}
$$

First, we find the error probability of the $S-D$ link under the condition that the instantaneous SNR of the $S-D$ link is higher than the instantaneous biased SNRs of the $R_{k}-D$ links [10]. The conditional error probability of the $S-D$ link under the condition on the instantaneous SNR, i.e., $\gamma_{S D}$, can be calculated from (2). Averaging (2) over the probability density function (pdf) of $\gamma_{S D}$ under the condition that $\gamma_{S D} \geq \rho_{i} \gamma_{R_{i} D}, i=1,2, \ldots, K$, the average BER of the $S-D$ link can be written as 


$$
\begin{aligned}
& \mathrm{BER}_{\gamma_{S D} \geq \rho_{i} \gamma_{R_{i} D}} \\
& =\int_{\gamma_{S D}=0}^{\rho_{1}^{-1} \gamma_{S D}} \int_{\gamma_{R_{1} D}=0}^{\rho_{K}^{-1} \gamma_{S D}} \int_{\gamma_{R_{K} D}=0}^{\gamma_{M_{s}} Q} c_{\left(\sqrt{2 d_{M_{s}}^{2} \gamma_{S D}}\right)} \times\left[\frac{1}{\bar{\gamma}_{S D}} e^{-\frac{\gamma_{S D}}{\bar{\gamma}_{S D}}}\left[\prod_{i=1}^{K} \frac{1}{\bar{\gamma}_{R_{i} D}} e^{-\frac{\gamma_{R_{i} D}}{\bar{\gamma}_{R_{i} D}}}\right]\right] d_{\gamma_{S D}} d_{\gamma_{R_{1} D}} \ldots d_{\gamma_{R_{K} D}} .
\end{aligned}
$$

Simplifying (9), we can write

$$
\begin{aligned}
& \mathrm{BER}_{\gamma_{S D} \geq \rho_{i} \gamma_{R_{i} D}} \\
& =\int_{\gamma_{S D}=0}^{\infty} c_{M_{s}} Q\left(\sqrt{2 d_{M_{s}}^{2} \gamma_{S D}}\right)\left[\frac{1}{\bar{\gamma}_{S D}} e^{-\frac{\gamma_{S D}}{\bar{\gamma}_{S D}}}\right] \\
& \times\left[1+\sum_{k=1}^{K} \sum_{y=1}^{\left(\begin{array}{c}
K \\
k
\end{array}\right)}(-1)^{k} e^{\left(-\gamma_{S D}\right) \frac{H M\left\{P_{k, y}(\mathcal{S})\right\}}{k}}\right] d_{\gamma_{S D},}
\end{aligned}
$$

where $H M\{$.$\} represents the harmonic mean; the set is defined$ as $\mathcal{S}=\left\{\rho_{i} \bar{\gamma}_{R_{i} D}\right\}, \mathrm{i}=1,2, \ldots, K$, and $P_{k, y}(\mathcal{S})$ is the $y$-th element of the $k$-element power set of $\mathcal{S}$.

Solving (10), we obtain

$$
\begin{aligned}
& \operatorname{BER}_{\gamma_{S D} \geq \rho_{i} \gamma_{R_{i}}}=I\left(c_{M_{s}}, d_{M_{s}}^{2}, \bar{\gamma}_{S D}\right) \\
& +\sum_{k=1}^{K} \sum_{y=1}^{\left(\begin{array}{c}
K \\
k
\end{array}\right)}(-1)^{k}\left[\left(\frac{k+1}{H M\left\{\bar{\gamma}_{S D}, P_{k, y}(\mathcal{S})\right\}}\right)\right. \\
& \left.\times I\left(\frac{c_{M_{s}}}{\bar{\gamma}_{S D}}, d_{M_{s}}^{2}, \frac{k+1}{H M\left\{\bar{\gamma}_{S D}, P_{k, y}(\mathcal{S})\right\}}\right)\right] .
\end{aligned}
$$

In the above, we used the following function:

$$
\begin{aligned}
I(a, b, c) & =\int_{0}^{\infty} a Q(\sqrt{2 b t}) \frac{1}{c} e^{-\frac{t}{c}} d_{t} \\
& =0.5 a\left(1-\sqrt{\frac{b c}{1+b c}}\right) .
\end{aligned}
$$

For example, the average BER of the $S-D$ link in the two-relay scenario is given by (13) at the top of the next page.

Next, we find the error probability of the $R_{i}-D$ link under the condition that the instantaneous biased SNR of the $R_{i}-D$ link is higher than the instantaneous SNR of the $S-D$ link and the instantaneous SNR of any other $R_{k}-D$ link. The conditional error probability of the $R_{i}-D$ link under the condition on the instantaneous SNR, i.e., $\gamma_{R_{i} D}$, can be obtained from (2). Averaging (2) over the pdf of $\gamma_{R_{i} D}$ under the condition that $\gamma_{S D}<\rho_{i} \gamma_{R_{i} D}$ and $\gamma_{R_{j} D}<\beta_{i j} \gamma_{R_{i} D}, j \neq$ $i, i, j=1,2, \ldots, K$, the average BER of the $R_{i}-D$ link is obtained by

$$
\begin{aligned}
& \mathrm{BER}_{\gamma_{S D}<\rho_{i} \gamma_{R_{i} D} \text { and } \gamma_{R_{j} D}<\beta_{i j} \gamma_{R_{i} D}} \\
& =\int_{\gamma_{R_{i} D}=0}^{\infty} \int_{\gamma_{S D}=0}^{\rho_{i} \gamma_{R_{i} D}} \int_{\gamma_{R_{1} D}=0}^{\beta_{i 1} \gamma_{R_{1} D}} \ldots \int_{\gamma_{R_{K} D}=0}^{\beta_{i K} \gamma_{R_{i} D}} c_{M_{R_{i}}} Q\left(\sqrt{2 d_{M_{R_{i}}}^{2} \gamma_{R_{i} D}}\right) \\
& \times\left[\frac{1}{\bar{\gamma}_{R_{i} D}} e^{-\frac{\gamma_{R_{i} D}}{\bar{\gamma}_{R_{i} D}}}\right]\left[\frac{1}{\bar{\gamma}_{S D}} e^{-\frac{\gamma_{S D}}{\bar{\gamma}_{S D}}}\right] \\
& \times\left[\prod_{j=1}^{K-1} \frac{1}{\bar{\gamma}_{R_{j} D}} e^{-\frac{\gamma_{R j D}}{\bar{\gamma}_{R_{j} D}}}\right] d_{\gamma_{R_{i} D}} d_{\gamma_{S D}} d_{\gamma_{R_{1} D}} \ldots d_{\gamma_{R_{K} D}} .
\end{aligned}
$$

Simplifying (14), we get

$$
\begin{aligned}
& \operatorname{BER}_{\gamma_{S D}<\rho_{i} \gamma_{R_{i} D} \text { and } \gamma_{R_{j} D}<\beta_{i j} \gamma_{R_{i} D} D} \\
& =\int_{\gamma_{R_{i} D}=0}^{\infty} c_{M_{R_{i}}} Q\left(\sqrt{2 d_{M_{R_{i}}}^{2} \gamma_{R_{i} D}}\right)\left[\frac{1}{\bar{\gamma}_{R_{i} D}} e^{-\frac{\gamma_{R_{i} D}}{\bar{\gamma}_{R_{i} D}}}\right] \\
& \times\left[1+\sum_{k=1}^{K} \sum_{y=1}^{\left(\begin{array}{l}
K \\
k
\end{array}\right)}(-1)^{k} e^{\left(-\gamma_{R_{i} D}\right) \frac{H M\left\{P_{k, y}\left(\mathcal{S}_{x}\right)\right\}}{k}}\right] d_{\gamma_{R_{i} D}},
\end{aligned}
$$

where $H M\{$.$\} represents the harmonic mean; the set is defined$ as $\mathcal{S}_{x}=\left\{\bar{\gamma}_{S D} \rho_{i}^{-1}, \bar{\gamma}_{R_{j} D} \beta_{i j}^{-1}\right\}, j \neq i, i, j=1,2, \ldots, K$, $P_{k, y}\left(\mathcal{S}_{x}\right)$ is the $y$-th element of the $k$-element power set of $\mathcal{S}_{x}$.

Solving (15), we obtain

$$
\begin{aligned}
& \operatorname{BER}_{\gamma_{S D}<\rho_{i} \gamma_{R_{i} D} \text { and } \gamma_{R_{j} D}<\beta_{i j} \gamma_{R_{i} D}}=I\left(c_{M_{R_{i}}}, d_{M_{R_{i}}}^{2}, \bar{\gamma}_{R_{i} D}\right) \\
& +\sum_{k=1}^{K} \sum_{y=1,2, \ldots, K}^{\left(\begin{array}{c}
K \\
k
\end{array}\right)}(-1)^{k}\left[\left(\frac{k+1}{H M\left\{\bar{\gamma}_{R_{i} D}, P_{k, y}\left(\mathcal{S}_{x}\right)\right\}}\right)\right. \\
& \left.\quad \times I\left(\frac{c_{M_{R_{i}}}}{\bar{\gamma}_{R_{i} D}}, d_{M_{R_{i}}}^{2}, \frac{k+1}{H M\left\{\bar{\gamma}_{R_{i} D}, P_{k, y}\left(\mathcal{S}_{x}\right)\right\}}\right)\right] .
\end{aligned}
$$

For example, the average BER of the $R_{1}-D$ link in the two-relay scenario is given by (17) in the next page.

Finally, using (10) and (16), BER $\mathrm{comp}_{\mathcal{D S}}$ can be expressed as (18) and (19):

$$
\begin{aligned}
& \mathrm{BER}_{\text {comp } p_{\mathcal{S}}}=\mathrm{BER}_{\gamma_{S D} \geq \rho_{1} \gamma_{R_{1} D}, \ldots, \rho_{K} \gamma_{R_{K} D}} \\
& +\sum_{i=1}^{K} \mathrm{BER}_{\gamma_{S D}<\rho_{i} \gamma_{R_{i} D} \text { and } \gamma_{R_{j} D}<\beta_{i j} \gamma_{R_{i} D}}{ }_{j \neq i, j=1,2, \ldots, K}
\end{aligned}
$$

\section{B. Finding the average BER}

By substituting (6), (7), and (19), in (4), the final expression for the average BER can be expressed by (20) given in the next page.

\section{Asymptotic Performance Analysis}

In this section, we focus on the high SNR regime and derive an asymptotic BER expression for BER-based selection scheme 


$$
\begin{aligned}
& \operatorname{BER}_{\gamma_{S D} \geq \rho_{i} \gamma_{R_{i} D}}=I\left(c_{M_{s}}, d_{M_{s}}^{2}, \bar{\gamma}_{S D}\right)-\left(\frac{1}{\frac{1}{\bar{\gamma}_{S D}}+\frac{\rho_{1}^{-1}}{\bar{\gamma}_{R_{1} D}}}\right) I\left(\frac{c_{M_{s}}}{\bar{\gamma}_{S D}}, d_{M_{s}}^{2}, \frac{1}{\frac{1}{\overline{\bar{\gamma}_{S D}}}+\frac{\rho_{1}^{-1}}{\bar{\gamma}_{R_{1} D}}}\right) \\
&-\left(\frac{1}{\frac{1}{\bar{\gamma}_{S D}}+\frac{\rho_{2}^{-1}}{\bar{\gamma}_{R_{2} D}}}\right) I\left(\frac{c_{M_{s}}}{\bar{\gamma}_{S D}}, d_{M_{s}}^{2}, \frac{1}{\frac{1}{\bar{\gamma}_{S D}}+\frac{\rho_{2}^{-1}}{\bar{\gamma}_{R_{2} D}}}\right)+\left(\frac{1}{\frac{1}{\bar{\gamma}_{S D}}+\frac{\rho_{1}^{-1}}{\bar{\gamma}_{R_{1} D}}+\frac{\rho_{2}^{-1}}{\bar{\gamma}_{R_{2} D}}}\right) I\left(\frac{c_{M_{s}}}{\bar{\gamma}_{S D}}, d_{M_{s}}^{2}, \frac{1}{\frac{1}{\bar{\gamma}_{S D}}+\frac{\rho_{1}^{-1}}{\bar{\gamma}_{R_{1} D}}+\frac{\rho_{2}^{-1}}{\bar{\gamma}_{R_{2} D}}}\right) .
\end{aligned}
$$

$$
\begin{aligned}
& \operatorname{BER}_{\gamma_{S D}<\rho_{1} \gamma_{R_{1} D} \text { and } \gamma_{R_{j} D}<\beta_{1 j} \gamma_{R_{1} D}}=I\left(c_{M_{R_{1}}}, d_{M_{R_{1}}}^{2}, \bar{\gamma}_{R_{1} D}\right)-\left(\frac{1}{\frac{1}{\bar{\gamma}_{R_{1} D}}+\frac{\rho_{1}}{\bar{\gamma}_{S D}}}\right) I\left(\frac{c_{M_{R_{1}}}}{\bar{\gamma}_{R_{1} D}}, d_{M_{R_{1}}}^{2}, \frac{1}{\frac{1}{\bar{\gamma}_{R_{1} D}}+\frac{\rho_{1}}{\overline{\gamma_{S} D}}}\right) \\
& -\left(\frac{1}{\frac{1}{\overline{\gamma_{R_{1}} D}}+\frac{\beta_{12}}{\bar{\gamma}_{R_{2} D}}}\right) I\left(\frac{c_{M_{R_{1}}}}{\bar{\gamma}_{R_{1} D}}, d_{M_{R_{1}}}^{2}, \frac{1}{\frac{1}{\bar{\gamma}_{R_{1} D}}+\frac{\beta_{12}}{\bar{\gamma}_{R_{2} D}}}\right)+\left(\frac{1}{\frac{1}{\overline{\bar{\gamma}_{R_{1} D}}}+\frac{\rho_{1}}{\bar{\gamma}_{S D}}+\frac{\beta_{12}}{\bar{\gamma}_{R_{2} D}}}\right) I\left(\frac{c_{M_{R_{1}}}}{\bar{\gamma}_{R_{1} D}}, d_{M_{R_{1}}}^{2}, \frac{1}{\frac{1}{\bar{\gamma}_{R_{1} D}}+\frac{\rho_{1}}{\bar{\gamma}_{S D}}+\frac{\beta_{12}}{\bar{\gamma}_{R_{2} D}}}\right) \text {. }
\end{aligned}
$$

$$
\begin{aligned}
& \text { BER }{\text { comp } p_{\mathcal{S}}}=I\left(c_{M_{s}}, d_{M_{s}}^{2}, \bar{\gamma}_{S D}\right)+\sum_{k=1}^{K} \sum_{y=1}^{\left(\begin{array}{c}
K \\
k
\end{array}\right)}(-1)^{k} I\left(\frac{c_{M_{s}}}{\bar{\gamma}_{S D}}, d_{M_{s}}^{2}, \frac{k+1}{H M\left\{\bar{\gamma}_{S D}, P_{k, y}(\mathcal{S})\right\}}\right)\left(\frac{k+1}{H M\left\{\bar{\gamma}_{S D}, P_{k, y}(\mathcal{S})\right\}}\right) \\
& +\sum_{i=1}^{K}\left[I\left(c_{M_{R_{i}}}, d_{M_{R_{i}}}^{2}, \bar{\gamma}_{R_{i} D}\right)+\sum_{k=1}^{K} \sum_{y=1}^{\left(\begin{array}{l}
K \\
k
\end{array}\right)}(-1)^{k} I\left(\frac{c_{M_{R_{i}}}}{\bar{\gamma}_{R_{i} D}}, d_{M_{R_{i}}}^{2}, \frac{k+1}{H M\left\{\bar{\gamma}_{R_{i} D}, P_{k, y}\left(\mathcal{S}_{x}\right)\right\}}\right)\left(\frac{k+1}{H M\left\{\bar{\gamma}_{R_{i} D}, P_{k, y}\left(\mathcal{S}_{x}\right)\right\}}\right)\right] .
\end{aligned}
$$

$$
\begin{aligned}
& \mathrm{BER}=\left(\prod_{k=1}^{K}\left[1-\left(1-\frac{1}{2} c_{M_{s}} \log _{2}\left(M_{s}\right)\left(1-\sqrt{\frac{d_{M_{s}}^{2} \bar{\gamma}_{S R_{k}}}{1+d_{M_{s}}^{2} \bar{\gamma}_{S R_{k}}}}\right)\right)^{\frac{N}{\log _{2} M_{s}}}\right]\right)\left(\frac{1}{2} c_{M_{S}}\left(1-\sqrt{\frac{d_{M_{S}}^{2} \bar{\gamma}_{S D}}{1+d_{M_{S}}^{2} \bar{\gamma}_{S D}}}\right)\right) \\
& +\sum_{r=1}^{K} \sum_{m=1}^{\left|P_{r}\left(S_{\text {all }}\right)\right|}\left[\prod_{e_{i} \in P_{r, m}\left(S_{\text {all }}\right)}\left(\left(1-\frac{1}{2} c_{M_{s}} \log _{2}\left(M_{s}\right)\left(1-\sqrt{\frac{d_{M_{s}}^{2} \bar{\gamma}_{S R_{e_{i}}}}{1+d_{M_{s}}^{2} \bar{\gamma}_{S R_{e_{i}}}}}\right)\right)^{\frac{N}{\log _{2} M_{s}}}\right)\right. \\
& \times \prod_{e_{o} \notin P_{r, m}\left(S_{\text {all }}\right)}\left(1-\left(1-\frac{1}{2} c_{M_{s}} \log _{2}\left(M_{s}\right)\left(1-\sqrt{\frac{d_{M_{s}}^{2} \bar{\gamma}_{S R_{e_{o}}}}{1+d_{M_{s}}^{2} \bar{\gamma}_{S R_{e_{o}}}}}\right)\right)^{\frac{N}{\log _{2} M_{s}}}\right) \\
& \times\left[I\left(c_{M_{s}}, d_{M_{s}}^{2}, \bar{\gamma}_{S D}\right)+\sum_{k=1}^{K} \sum_{y=1}^{\left(\begin{array}{c}
K \\
k
\end{array}\right)}(-1)^{k} I\left(\frac{c_{M_{s}}}{\bar{\gamma}_{S D}}, d_{M_{s}}^{2}, \frac{k+1}{H M\left\{\bar{\gamma}_{S D}, P_{k, y}(\mathcal{S})\right\}}\right)\left(\frac{k+1}{H M\left\{\bar{\gamma}_{S D}, P_{k, y}(\mathcal{S})\right\}}\right)\right. \\
& \left.+\sum_{i=1}^{K}\left[I\left(c_{M_{R_{i}}}, d_{M_{R_{i}}}^{2}, \bar{\gamma}_{R_{i} D}\right)+\sum_{k=1}^{K} \sum_{y=1}^{\left(\begin{array}{c}
K \\
k
\end{array}\right)}(-1)^{k} I\left(\frac{c_{M_{R_{i}}}}{\bar{\gamma}_{R_{i} D}}, d_{M_{R_{i}}}^{2}, \frac{k+1}{H M\left\{\bar{\gamma}_{R_{i} D}, P_{k, y}\left(\mathcal{S}_{x}\right)\right\}}\right)\left(\frac{k+1}{H M\left\{\bar{\gamma}_{R_{i} D}, P_{k, y}\left(\mathcal{S}_{x}\right)\right\}}\right)\right]\right] .
\end{aligned}
$$

as SNR goes to the infinity in order to obtain further insight into the performance of the system. The asymptotic approximations for $\mathrm{PER}_{S R_{i}}$ and $\mathrm{BER}_{S D}$ are found in [7] when the average SNRs are expressed as $\bar{\gamma}_{S D}=\sigma_{S D}^{2} \mathrm{SNR}, \bar{\gamma}_{S R}=\sigma_{S R}^{2} \mathrm{SNR}$,

$$
\mathrm{PER}_{S R_{i}} \stackrel{\mathrm{SNR} \rightarrow \infty}{\approx} \frac{N c_{M_{S}}}{4 d_{M_{S}}^{2} \sigma_{S R_{i}}^{2} \mathrm{SNR}}
$$
and $\bar{\gamma}_{R D}=\sigma_{R D}^{2}$ SNR:

$$
\mathrm{BER}_{S D} \stackrel{\mathrm{SNR} \rightarrow \infty}{\approx} \frac{c_{M_{S}}}{4 d_{M_{S}}^{2} \sigma_{S D}^{2} \mathrm{SNR}}
$$




$$
\left(1-\mathrm{PER}_{S R_{i}}\right) \stackrel{\mathrm{SNR} \rightarrow \infty}{\approx} 1-\frac{N c_{M_{S}}}{4 d_{M_{S}}^{2} \sigma_{S R_{i}}^{2} \mathrm{SNR}} \stackrel{\mathrm{SNR} \rightarrow \infty}{\approx} 1 .
$$

Then, for the case that the instantaneous SNR of the $S-D$ link is higher than the instantaneous SNRs of the relay-todestination links,

$$
\begin{aligned}
& \operatorname{BER}_{\gamma_{S D} \geq \rho_{i} \gamma_{R_{i} D}} \\
& \stackrel{\mathrm{SNR} \rightarrow \infty}{=} \int_{\gamma_{S D}=0}^{\infty} \int_{\gamma_{R_{1} D}=0}^{\rho_{1}^{-1} \gamma_{S D}} \cdots \int_{\gamma_{R_{2} D}=0}^{\rho_{K}^{-1} \gamma_{S D}} c_{M_{s}} Q\left(\sqrt{2 d_{M_{s}}^{2} \gamma_{S D}}\right) \\
& \times \frac{1}{\overline{\gamma_{S D}}}\left[\prod_{i=1}^{K} \frac{1}{\bar{\gamma}_{R_{i} D}}\right] d_{\gamma_{S D}} d_{\gamma_{R_{1} D}} \ldots d_{\gamma_{R_{K} D}} \\
& =\frac{c_{M_{s}}}{2 \bar{\gamma}_{S D}}\left[\prod_{i=1}^{K} \frac{\rho_{i}^{-1}}{\bar{\gamma}_{R_{i} D}}\right] \int_{\gamma_{S D}=0}^{\infty} \operatorname{erfc}\left(\sqrt{d_{M_{R}}^{2} \gamma_{R D}}\right) \gamma_{S D}^{K} d_{\gamma_{S D}} \\
& =\left[\prod_{i=1}^{K} \frac{\rho_{i}^{-1}}{\bar{\gamma}_{R_{i} D}}\right] \frac{c_{M_{s}} \Gamma(K+1.5)}{2 \sqrt{\pi} \bar{\gamma}_{S D}(1+K)\left(d_{M_{s}}^{2}\right)^{K+1}} .
\end{aligned}
$$

Also, for the case that the instantaneous SNR of the $R_{i}-D$ is higher than the instantaneous SNR of the $S-D$ link and instantaneous SNR of any other $R_{k}-D$ link,

$\mathrm{BER}_{\gamma_{S D}<\rho_{i} \gamma_{R_{i} D} \text { and } \gamma_{R_{j} D}<\beta_{i j} \gamma_{R_{i} D}}$
$\stackrel{\mathrm{SNR} \rightarrow \infty, j=1,2, \ldots, K}{=} \sum_{i=1}^{K}\left[\prod_{\substack{j=1 \\ j \neq i}}^{K} \frac{\beta_{i j}}{\bar{\gamma}_{R_{j} D}}\right] \frac{\rho_{i} c_{M_{i}} \Gamma(K+1.5)}{2 \sqrt{\pi} \bar{\gamma}_{S D} \bar{\gamma}_{R_{i} D}(1+K)\left(d_{M_{i}}^{2}\right)^{K+1}}$.

By substituting (24) and (25) in (18), we get (26).

$$
\begin{aligned}
& \mathrm{BER}_{\text {comp }_{\mathcal{D S}}} \\
& \stackrel{\mathrm{SNR} \rightarrow \infty}{=}\left[\left[\prod_{i=1}^{K} \frac{\rho_{i}^{-1}}{\sigma_{R_{i} D}^{2}}\right] \frac{c_{M_{s}} \Gamma(K+1.5)}{2 \sqrt{\pi} \sigma_{S D}^{2}(1+K)\left(d_{M_{s}}^{2}\right)^{K+1}}\right. \\
& +\sum_{i=1}^{K} \frac{\rho_{i} c_{M_{i}} \Gamma(K+1.5)}{2 \sqrt{\pi} \sigma_{S D}^{2} \sigma_{R_{i} D}^{2}(1+K)\left(d_{M_{i}}^{2}\right)^{K+1}} \\
& \left.\times\left[\prod_{\substack{j=1 \\
j \neq i}}^{K} \frac{\beta_{i j}}{\sigma_{R_{j} D}^{2}}\right]\right] \frac{1}{\mathrm{SNR}^{K+1}} .
\end{aligned}
$$

Finally, by substituting (21), (22), (23), and (26), in (4), we obtain (27) at the top of the next page.

For example, for the two-relay scenario, the asymptotic approximation is given by (28) given in the next page.

\section{Simulation Results}

In this section, we investigate the performance of selective relaying with different modulation levels in a multi-relay scenario through Monte-Carlo simulation. In Fig. 1, we show

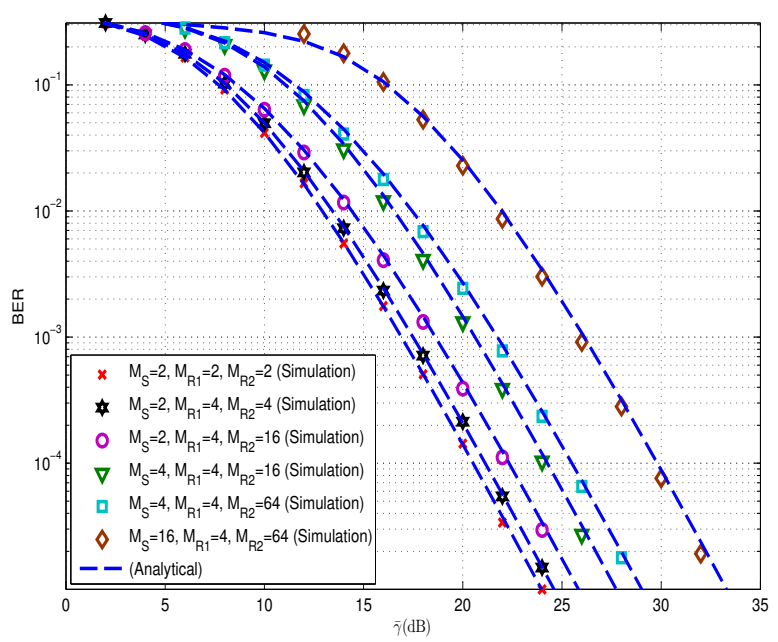

Fig. 1. BER performance of BER-based selection scheme for two relay scenario, assuming $\bar{\gamma}_{S R_{1}}=\bar{\gamma}+10, \bar{\gamma}_{S R_{2}}=\bar{\gamma}+10, \bar{\gamma}_{S D}=\bar{\gamma}-10$, $\bar{\gamma}_{R_{1} D}=\bar{\gamma}, \bar{\gamma}_{R_{2} D}=\bar{\gamma}$ and $N=264$ bits. It is clear from the figures that the derived BER expressions given by (20) and the simulation results are in excellent agreement.

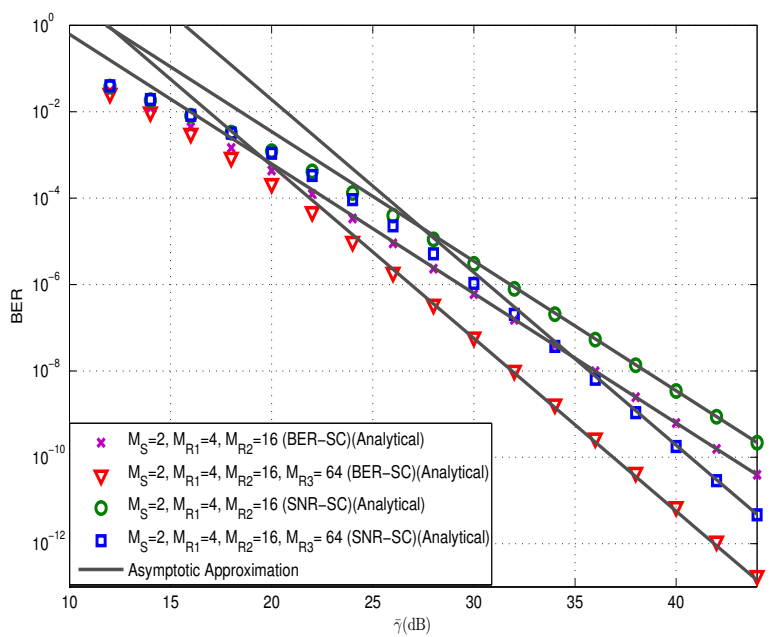

Fig. 2. Asymptotic BER performance of BER-based selection scheme and SNR-based selection scheme for two and three relay scenarios, assuming $\bar{\gamma}_{S R_{1}}=\bar{\gamma}+10, \bar{\gamma}_{S R_{2}}=\bar{\gamma}+10, \bar{\gamma}_{S R_{3}}=\bar{\gamma}+10, \bar{\gamma}_{S D}=\bar{\gamma}-10$, $\bar{\gamma}_{R_{1} D}=\bar{\gamma}, \bar{\gamma}_{R_{2} D}=\bar{\gamma}, \bar{\gamma}_{R_{3} D}=\bar{\gamma}$ and $N=264$ bits.

the BER simulation results for the BER-based selection scheme in the two relay scenario. It is clear from the figure that the derived analytical results are in excellent agreement with the simulation results. In Fig. 2, we plot the asymptotic BER expressions for BER-based selection scheme and SNR-based selection scheme for different number of relay nodes. We confirm that the asymptotic expression is tight for high SNRs. Although both BER-based selection scheme and SNR-based selection scheme achieve the same diversity order, BER-based selection scheme achieves higher SNR gain for all cases. 


$$
\begin{aligned}
& \mathrm{BER}^{\mathrm{SNR} \rightarrow \infty}=\left(\prod_{k=1}^{K} \frac{N c_{M_{S}}}{4 d_{M_{S}}^{2} \sigma_{S R_{k}}^{2}}\right) \frac{c_{M_{S}}}{4 d_{M_{S}}^{2} \sigma_{S D}^{2}} \frac{1}{\mathrm{SNR}^{K+1}}+\sum_{r=1}^{K} \sum_{m=1}^{\left|P_{r}\left(\mathcal{S}_{\text {all }}\right)\right|}\left(\prod_{e_{o} \notin P_{r, m}\left(\mathcal{S}_{a l l}\right)} \frac{N c_{M_{S}}}{4 d_{M_{S}}^{2} \sigma_{S R_{e_{o}}}^{2} \mathrm{SNR}}\right) \\
& \times\left[\left[\prod_{i=1}^{\left|P_{r, m}\left(\mathcal{S}_{\text {all }}\right)\right|} \frac{\rho_{i}^{-1}}{\sigma_{R_{i} D}^{2}}\right] \frac{c_{M_{s}} \Gamma\left(\left|P_{r, m}\left(\mathcal{S}_{\text {all }}\right)\right|+1.5\right)}{2 \sqrt{\pi} \sigma_{S D}^{2}\left(1+\left|P_{r, m}\left(\mathcal{S}_{\text {all }}\right)\right|\right)\left(d_{M_{s}}^{2}\right)^{\left|P_{r, m}\left(\mathcal{S}_{\text {all }}\right)\right|+1}} \frac{1}{\operatorname{SNR}\left|P_{r, m}\left(\mathcal{S}_{\text {all }}\right)\right|+1}\right. \\
& \left.+\sum_{i=1}^{\left|P_{r, m}\left(S_{a l l}\right)\right|}\left[\prod_{\substack{j=1 \\
j \neq i}}^{\left|P_{r, m}\left(\mathcal{S}_{\text {all }}\right)\right|} \frac{\beta_{i j}}{\sigma_{R_{j} D}^{2}}\right] \frac{\rho_{i} c_{M_{i}} \Gamma\left(\left|P_{r, m}\left(\mathcal{S}_{\text {all }}\right)\right|+1.5\right)}{2 \sqrt{\pi} \sigma_{S D}^{2} \sigma_{R_{i} D}^{2}\left(1+\left|P_{r, m}\left(\mathcal{S}_{a l l}\right)\right|\right)\left(d_{M_{i}}^{2}\right)^{\left|P_{r, m}\left(\mathcal{S}_{a l l}\right)\right|+1}} \frac{1}{\operatorname{SNR}^{\left|P_{r, m}\left(\mathcal{S}_{a l l}\right)\right|+1}}\right] \text {. } \\
& \mathrm{BER} \stackrel{\mathrm{SNR} \rightarrow \infty}{=}\left[\frac{N^{2} c_{M_{S}}^{3}}{64 d_{M_{S}}^{6} \sigma_{S R_{1}}^{2} \sigma_{S R_{2}}^{2} \sigma_{S D}^{2}}+\frac{\Gamma(2.5) N c_{M_{S}}\left(\rho_{2}^{-1} c_{M_{s}} d_{M_{R_{2}}}^{4}+\rho_{2} c_{M_{R_{2}}} d_{M_{s}}^{4}\right)}{16 \sqrt{\pi} d_{M_{R_{2}}}^{4} d_{M_{s}}^{6} \sigma_{S R_{1}}^{2} \sigma_{S D}^{2} \sigma_{R_{2} D}^{2}}+\frac{\Gamma(2.5) N c_{M_{S}}\left(\rho_{1}^{-1} c_{M_{s}} d_{M_{R_{1}}}^{4}+\rho_{1} c_{M_{R_{1}}} d_{M_{s}}^{4}\right)}{16 \sqrt{\pi} d_{M_{R_{1}}}^{4} d_{M_{s}}^{6} \sigma_{S R_{2}}^{2} \sigma_{S D}^{2} \sigma_{R_{1} D}^{2}}\right. \\
& \left.+\frac{\Gamma(3.5)\left(\rho_{1}^{-1} \rho_{2}^{-1} c_{M_{s}} d_{M_{R_{2}}}^{6} d_{M_{R_{1}}}^{6}+\rho_{1} \beta_{12} c_{M_{R_{1}}} d_{M_{s}}^{6} d_{M_{R_{2}}}^{6}+\rho_{2} \beta_{21} c_{M_{R_{2}}} d_{M_{s}}^{6} d_{M_{R_{1}}}^{6}\right)}{6 \sqrt{\pi} d_{M_{R_{1}}}^{6} d_{M_{R_{2}}}^{6} d_{M_{s}}^{6} \sigma_{R_{1} D}^{2} \sigma_{S D}^{2} \sigma_{R_{2} D}^{2}}\right] \frac{1}{\mathrm{SNR}^{3}} .
\end{aligned}
$$

\section{CONCLUSIONS}

With the advent of the more advanced wireless access network architectures, previously unencountered physical layer scenarios emerge. In this paper, we studied one such scenario, in which the destination identifies the best link among a set of possible links ("best" in terms of minimizing the BER) under the general setting that those links are allowed to use different modulation levels (to take advantage of the link quality). This problem set up may correspond to a number of different architectures as highlighted in the Abstract and Introduction; without loss of generality, we considered a multi-relay selective diversity scheme as the context of this paper.

The main contribution of this paper is the derivation an analytical expression for the e2e uncoded BER performance. We also provided a simpler approximate BER expression that is accurate in the high SNR regime. Monte-Carlo simulations are provided to validate the analytical derivations.

The BER results presented in this paper can be utilized towards determining a number of important parameters in the operation of the network; for instance, for a given link quality (SNR), the modulation level to be used that will maximize the e2e spectral efficiency under the selective relaying framework.

\section{REFERENCES}

[1] A. Bletsas, A. Khisti, D. P. Reed, and A. Lippman, "A simple cooperative diversity method based on network path selection," IEEE J. Select. Areas Commun., vol. 24, no. 3, pp. 659-672, 2006.

[2] K. M. Thilina and E. Hossain, "Selective relaying in multi-relay networks with feedback delays and adaptive modulation," in Proc. IEEE Int. Conf. Commun. (ICC), 2012, pp. 4171-4175.

[3] S. Hares, H. Yanikomeroglu, and B. Hashem, "Diversity and AMC (adaptive modulation and coding)-aware routing in TDMA multihop networks," in Proc. IEEE Glob. Commun. Conf. (Globecom), vol. 1, 2003 , pp. 458-463.

[4] S. Valentin, D. H. Woldegebreal, T. Volkhausen, and H. Karl, "Combining for cooperative WLANs - a reality check based on prototype measurements," in Proc. IEEE Int. Conf. Commun. (ICC) Wkshp., 2009, pp. 1-5.

[5] Y. Zhang, Y. Ma, and R. Tafazolli, "Modulation-adaptive cooperation schemes for wireless networks," in Proc. IEEE Veh. Tech. Conf. (VTCSpring), 2008, pp. 1320-1324.
[6] A. Bin Sediq and H. Yanikomeroglu, "Performance analysis of soft-bit maximal ratio combining in cooperative relay networks," IEEE Trans. Wireless Commun., vol. 8, no. 10, pp. 4934-4939, 2009.

[7] _ - "Performance analysis of selection combining of signals with different modulation levels in cooperative communications," IEEE Trans. Veh. Technol., vol. 60, no. 4, pp. 1880-1887, 2011.

[8] - "Selection combining of signals with different modulation levels in Nakagami-m fading," IEEE Commun. Lett., vol. 16, no. 5, pp. 752-755, 2012.

[9] B. Sklar, Digital Communications, 2nd ed. Prentice Hall, 2001.

[10] M. Selvaraj and R. K. Mallik, "Scaled selection combining based cooperative diversity system with decode and forward relaying," IEEE Trans. Veh. Technol., vol. 59, no. 9, pp. 4388-4399, 2010. 